\title{
Critical Fragility Analysis for Electro-Mechanical System under Harsh Operating Conditions
}

\author{
Qiang Sun, Jiguang Yue, Yanming Wang, Yongqing Su and Yanchao Dong* \\ College of Electronics and Information Engineering, Tongji University, \\ No. 4800 Cao'an Highway, 201804 Shanghai, P.R. China \\ Corresponding Author's E-mail: dongyanchao@tongji.edu.cn
}

\begin{abstract}
For industrial electro-mechanical systems, the compromised design might result in drastically performance degradation under unexpected, sudden operating condition changes, and the possible degradation is considered as the fragility of industrial electromechanical systems in this paper. A minimum system performance index is defined and with the index corresponding the definition of critical fragile status of the system is introduced. This paper discusses the identification of interval system model under harsh operating conditions, uses the frequency response stable margin as the critical fragile status criterion, and proposes a sub-optimal multi-group iteration interval branch-andbound algorithm to search the critical status. Finally, an engineering experiment based on the low temperature tests of a direct drive pump controlled (DDPC) position servo system is given to verify the effectiveness of the proposed method.
\end{abstract}

Keywords: critical fragile status, harsh condition, branch-and-bound, direct drive pump controlled system

\section{Introduction}

Fragility is a usually mentioned concept in researches of control systems in recent years, however a shared understanding of fragility has not formed yet. Keel et al. [1] argued that fragility is the loss of stability and robustness in a well-designed system due to small perturbations and concluded that poor controller design results in fragility. Mäkilä [2] questioned the conclusions of Keel, and suggested that the flaws in controller design could be solved by adopting sensible optimization criteria, and should not be considered to the cause of fragility. The afterward studies [3-5] of system fragility had emphasized the theoretical design process of the overall control system, and raised analysis methods utilizing Bode integrals and the Nyquist stable margin. Previous researches paid little attention of fragility analysis regarding the fragility analysis of realistic electro-mechanical systems.

It is a common sense that the industrial electro-mechanical control systems are designed with an acceptable range of operating conditions, and the robustness of the system guarantees the overall stability under certain perturbations. Sudden change of operating conditions, flaws of the facilities themselves result in the uncertainness of the control system, which may lead to performance decrement or even failure. The aforementioned performance degradation under certain harsh operating conditions is considered as the fragility of industrial electro-mechanical system in this paper. In most cases, the cost of the system would be greatly raised if any possible sudden harsh change in operating condition is considered. Due to the compromised design, the fragility should be considered as an intrinsic characteristic of industrial electro-mechanical systems, and it is crucial to discuss the fragility of the compromising-designed systems. In this paper,

* Corresponding author 
the operating condition, under which the system could not fulfill the performance index, is defined as critical fragile condition; and the corresponding system operating status is defined as the critical fragile status. If the system still operates under the critical fragile condition, the health status of the system itself might also be endangered. Thus the analysis of the critical fragile status of industrial systems has a positive effect to the health status monitoring and risk control of the systems.

Many of the industrial electro-mechanical systems have mature structures and clear operation schemes. Considering the acceptable errors in practical, some of the systems could even be simplified as linear minimum phase systems; therefore, with the aid of the transfer functions, the analysis of the systems would be greatly facilitated. However, due to the errors in the process of manufacturing, installing and adjusting of the systems, there are differences between the parameters in practical and nominal. Furthermore, some of the key parameters even vary when the operating condition changes. Moore [6] raised the conception of interval numbers, and set up the foundation of interval arithmetic. Interval numbers, which intrinsically reserve true values in representation itself and during computations, describe uncertainness with a more natural way which corresponds the thoughts of human. With interval numbers to describe the uncertainness of the system parameters, interval models could be a favorable way to represent the system model under a certain range of operating conditions.

Based on interval model and operating data, this paper proposes a method of searching critical fragile status of electro-mechanical system under harsh operating conditions. The criterion of critical fragile status is set as the system's maximum power drop by a certain amount compared to the standard status, which can be directly reflected into frequency response. The model corresponding to the critical fragile status is named as critical fragile model in this paper. During the key procedures of searching for critical model from an interval model, a sub-optimal method with engineering accuracy, namely a multi-group iterative interval branch-and-bound algorithm, is proposed in this paper. In the proposed algorithm, the overall estimating vector is divided into small vector groups with iteratively searching process so that the optimized results can be derived more efficiently compared with ordinary branch-and-bound method. The main procedure of establishing the critical model is as follow:

(1) Build the scheme model according to the operating and physical characteristics, and decide the order of the scheme model;

(2) Estimate system parameters in harsh environments using operating data under different operation conditions;

(3) Determine the criteria of critical fragile status of the system, according to the required power drop amount;

(4) Establish the interval model with combination of all separate models estimated from different operating conditions. Use the multi-group iterative interval branchand-bound algorithm to find the critical fragile status;

(5) Evaluate the fragility and possible maintenance mechanism according to the critical fragile status.

\section{Establishment of Interval System Model under Different Operating Conditions}

Parameters change when the actual operating status varies. One possible method of estimating parameters is the least square method [7]. With the zero load operation data under different operating conditions, the upper and lower boundary of each parameter interval could be estimated.

Set the position output series as $y(k)$, given signal series as $u(k)$, the order of system transfer function's denominator and numerator as $n_{\alpha}, n_{\beta}$, and the poles and zeros to be 
estimated in the discrete system could be written as a vector $\boldsymbol{\phi}=\left[\alpha_{1}, \alpha_{2}, \cdots, \alpha_{n_{\alpha}}, \gamma_{1}, \gamma_{2}, \cdots, \gamma_{n_{\beta}}\right]^{\mathrm{T}}$. Ignoring the measurement noises, the measurement model could be written as

$$
y(k)=\boldsymbol{h}^{\mathrm{T}}(k) \boldsymbol{\phi}
$$

, where $\boldsymbol{h}^{\mathrm{T}}(k)=\left[-y(k-1), \cdots,-y\left(k-n_{a}\right),-u(k-1)\right]$. Set the length of experiment data as $L\left(L>\left(n_{a}+n_{b}\right)\right)$, then (1) could be written as the following vector form

$$
\boldsymbol{Y}_{L}=\boldsymbol{H}_{L} \boldsymbol{\phi}
$$

The criterion function is chosen as $J(\phi)=\left(\boldsymbol{Y}_{L}-\boldsymbol{H}_{L} \boldsymbol{\phi}\right)^{\mathrm{T}}\left(\boldsymbol{Y}_{L}-\boldsymbol{H}_{L} \boldsymbol{\phi}\right)$, and the optimal estimation under the situation in which ${ }^{J\left(\boldsymbol{\theta}_{1}\right)}$ gets the minimum value could be drawn as:

$$
\hat{\boldsymbol{\phi}}=\left(\boldsymbol{H}_{L}^{\mathrm{T}} \boldsymbol{H}_{L}\right)^{-1} \boldsymbol{H}_{L}^{\mathrm{T}} \boldsymbol{Y}_{L}
$$

In summary, to set up the interval system model, 1) intercept $L$ groups of effective operation data under the same operating condition, and form the sample matrix $H_{L} ; 2$ ) estimate the parameters of the system by equation (3), and the discrete model is drawn; 3 ) according to zero-pole matching principle and the sampling time $T$ of the discrete system, replace $z$ with $e^{s T}$, then the continuous model is drawn; 4) identify the upper and lower boundary of the continuous model in the target range of operating conditions, and draw the interval system model in the s-plane as:

$$
G(s)=\frac{\left(s-\left[b_{1}\right]\right)\left(s-\left[b_{2}\right]\right) \cdots\left(s-\left[b_{n_{b}}\right]\right)}{\left(s-\left[a_{1}\right]\right)\left(s-\left[a_{2}\right]\right) \cdots\left(s-\left[a_{n_{a}}\right]\right)}
$$

, where $\left[a_{i}\right],\left[b_{j}\right], i=1,2, \cdots, n_{a}, j=1,2, \cdots, n_{b}$ represent the intervals of poles and zeros.

\section{Criterion for the Critical Fragile Status}

After the continuous model has been established, the frequency characteristics of the system are uniquely determined. System performance can be fully reflected by its frequency characteristics. According to the classic control theory, the bandwidth of a closed-loop system is usually defined as the frequency where the magnitude-frequency gain degrades by $3 \mathrm{~dB}$, i.e. the equivalent power drops to $50 \%$ of the maximum. In this paper, the criterion index of critical fragile status is set as the magnitude-frequency response curve whose magnitude gain is lower by $X \mathrm{~dB}$ (the value of $X$ could be set according to practical requirements) than the nominal worst operating condition curve at the bandwidth of the system under the nominal worst operating condition. When the system is a linear minimum phase system, the parameters of the critical model can be uniquely determined by the magnitude-frequency response. Set the magnitude-frequency response at $\omega$ as $20 \lg |G(\mathrm{j} \omega)|$, the bandwidth under nominal worst working condition as $\omega_{B 0}$. Therefore, the parameter estimation of the critical fragile model turns into the following optimization problem:

$$
\begin{aligned}
& \min E=|20 \lg | G\left(\mathrm{j} \omega_{B 0}\right)|+X| \\
& \text { s.t. } \boldsymbol{\theta} \in \boldsymbol{S}
\end{aligned}
$$

, where $\boldsymbol{S}$ is the feasible region of the estimating parameter vector $\boldsymbol{\theta}$. 


\section{Analysis of the Critical Fragile Status}

With the operation data collected under harsh operating conditions, an interval system model could be derived under the range of harsh conditions. The poles and zeros of the interval model form a series of intervals, or feasible ranges for the critical fragile status searching. In this section, a sub-optimal multi-group iterative interval branch-and-bound algorithm is discussed to search the critical fragile status using the frequency criterion.

The branch-and-bound algorithm [8-9] is a deterministic method to solve optimization problems. The algorithm dissects the original feasible region into small regions successively, and calculates the lower and upper boundary of the target function on each small region. With discarding the small regions whose lower boundary of the target function is larger than current upper boundary, the feasible region is reduced by iterations, then the algorithm stops after the feasible region shrinks into the allowed error range. One of the drawbacks of branch-and-bound algorithm is that the algorithm complexity would be significantly increased and the efficiency would be decreased obviously when the number of estimating parameters increases.

Considering the interval model (4), there might be conjugated poles or zeros, thus the total numbers of the estimating parameters is no more than $n_{a}+n_{b}$, and the estimating parameters could be arranged into groups due to the correlations. Therefore, in order to increase the algorithm efficiency, a sub-optimal multi-group iterative interval branchand-bound algorithm is proposed in this paper. Divide the elements of estimating parameter vector into $M$ groups $\left(M<n_{a}+n_{b}\right)$, i.e. $\boldsymbol{\theta}=\left[\boldsymbol{\theta}_{1}, \boldsymbol{\theta}_{2}, \cdots, \boldsymbol{\theta}_{M}\right]^{\mathrm{T}}$, where $\boldsymbol{\theta}_{\mathrm{i}}=\left[\eta_{1}, \eta_{2}, \cdots, \eta_{K}\right]^{\mathrm{T}}, K \leq n_{a}+n_{b}(i=1,2, \cdots, M)$; and each group corresponds its feasible region, i.e. the original feasible region could be divided into $M$ groups $\boldsymbol{t}=\left[\boldsymbol{S}_{1}, \boldsymbol{S}_{2}, \cdots, \boldsymbol{S}_{\mathrm{M}}\right]^{\mathrm{T}}$, where $\mathrm{S}_{i}=\left[\tau_{1}, \tau_{2}, \cdots, \tau_{K}\right]^{\mathrm{T}} . \eta_{k}(k=1,2, \cdots, K)$ is a single estimating parameter and interval number $\tau_{k}$ is the feasible region of $\eta_{k}$. Here $M$ sub-feasibleregion is constructed:

$$
\begin{gathered}
\boldsymbol{t}_{1}=\left[\boldsymbol{S}_{1},\left[\operatorname{mid}\left(\boldsymbol{S}_{2}\right), \operatorname{mid}\left(\boldsymbol{S}_{2}\right)\right], \cdots,\left[\operatorname{mid}\left(\boldsymbol{S}_{M}\right), \operatorname{mid}\left(\boldsymbol{S}_{M}\right)\right]\right]^{\mathrm{T}} \\
\boldsymbol{t}_{2}=\left[\left[\operatorname{mid}\left(\boldsymbol{S}_{1}\right), \operatorname{mid}\left(\boldsymbol{S}_{1}\right)\right], \boldsymbol{S}_{2}, \cdots,\left[\operatorname{mid}\left(\boldsymbol{S}_{M}\right), \operatorname{mid}\left(\boldsymbol{S}_{M}\right)\right]\right]^{\mathrm{T}} \\
\cdots \\
\boldsymbol{t}_{M}=\left[\left[\operatorname{mid}\left(\boldsymbol{S}_{1}\right), \operatorname{mid}\left(\boldsymbol{S}_{1}\right)\right],\left[\operatorname{mid}\left(\boldsymbol{S}_{2}\right), \operatorname{mid}\left(\boldsymbol{S}_{2}\right)\right], \cdots, \boldsymbol{S}_{M}\right]^{\mathrm{T}}
\end{gathered}
$$

Where $\operatorname{mid}(\cdot)$ means a middle value calculation. The optimal searching process using interval branch-and-bound algorithm is performed in each sub-feasible-region sequentially, which forms one step of the main iteration loop. The iteration formulas of sub-feasible-region are as below:

$$
\begin{gathered}
\boldsymbol{t}_{1}=\left[\boldsymbol{S}_{1},\left[\operatorname{mid}\left(\operatorname{mint}_{2}\{2\}\right), \operatorname{mid}\left(\operatorname{mint}_{2}\{2\}\right)\right], \cdots,\left[\operatorname{mid}\left(\min t_{M}\{M\}\right), \operatorname{mid}\left(\operatorname{mint}_{M}\{M\}\right)\right]\right]^{\mathrm{T}} \\
\boldsymbol{t}_{2}=\left[\left[\operatorname{mid}\left(\operatorname{mint}_{1}\{1\}\right), \operatorname{mid}\left(\operatorname{mint}_{1}\{1\}\right)\right], \boldsymbol{S}_{2}, \cdots,\left[\operatorname{mid}\left(\operatorname{mint}_{M}\{M\}\right), \operatorname{mid}\left(\min _{M}\{M\}\right)\right]\right]^{\mathrm{T}} \\
\cdots \\
\boldsymbol{t}_{M}=\left[\left[\operatorname{mid}\left(\operatorname{mint}_{1}\{1\}\right), \operatorname{mid}\left(\operatorname{mint}_{1}\{1\}\right)\right],\left[\operatorname{mid}\left(\operatorname{mint}_{2}\{2\}\right), \operatorname{mid}\left(\operatorname{mint}_{2}\{2\}\right)\right], \cdots, S_{M}\right]^{\mathrm{T}}
\end{gathered}
$$

, where $\min t_{i}\{i\}, i=1,2, \cdots, M$ refers to the $i^{\text {th }}$ element of $\min \boldsymbol{t}_{i}$ according to the grouping of $\boldsymbol{\theta}$. Finally, the iteration stops when the following condition is met:

$$
\sum_{i=2, j=i-1}^{M}\left(\operatorname{mid}\left(\min \boldsymbol{t}_{i}\right) \cdot-\boldsymbol{m i d}\left(\min \boldsymbol{t}_{j}\right)\right)^{2} \leq 0.1
$$


, where $\cdot-$ refers to the subdivision between elements. After the iteration stops, the parameters can be estimated sub-optimally. The structure of the whole algorithm is shown as Figure 1.

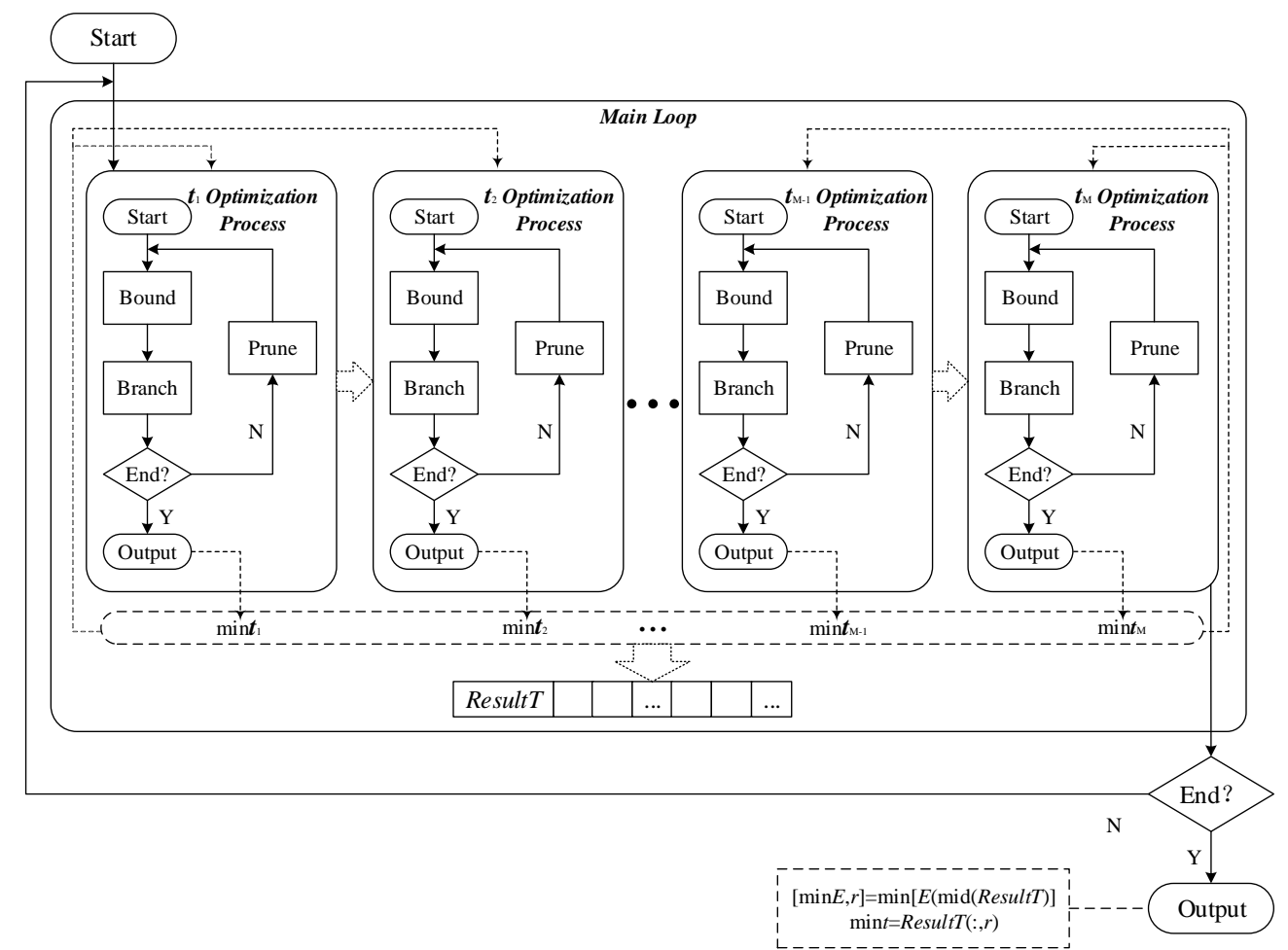

Figure 1. Structure of the Proposed Multi-Group Iterative Interval Branchand-Bound Algorithm

The detailed procedures of the multi-group iterative interval branch-and-bound algorithm are as follows:

(1) Divide the estimating parameters into $M$ groups by specified principles, and determine the original feasible region as $t=\left[S_{1}, S_{2}, \cdots, S_{n}\right]$.

(2) Initializations: set $\min \boldsymbol{t}_{i}=\boldsymbol{t}, i=1,2, \cdots, M$, and all sub-feasible-region.

(3) Main iteration loop:

1) Optimal searching process in sub-feasible-region $\boldsymbol{t}_{1}$ :

a) Pre-process: calculate the boundary $E$ of the target function, and determine the current minimum value $\min E$.

b) Initializations: set $n$ as the column number of ${ }^{t_{1}}$. Save the elements of $t_{1}$ into $t_{1}^{(1)}$, then clear $t_{1}$.

c) Branch: divide $\boldsymbol{t}_{1}^{(1)}$ into $N$ parts: consider the width of each elements in $\boldsymbol{t}_{1}^{(1)}$, dissect the widest element equally and keep other elements the same, then a new interval matrix $\boldsymbol{t}_{1}^{(2)}$ is formed.

d) Bound: determine the boundary of the target function in each small parts.

i. Calculate $E^{(2)}\left(\boldsymbol{t}_{1}^{(2)}\right)=20 \lg |G(\mathrm{j} \omega)|$.

ii. Determine the minimum value $\min E^{(2)}$ of the target function in the current step.

e) Prune: 
i. Move the columns in $t_{1}^{(2)}$ with lower width than 0.01 into $L t$, and the remaining columns forms $\boldsymbol{t}_{1}^{(3)}$ with the original order; The correspond columns in $E^{(2)}$ forms $L E$ and $E^{(3)}$.

ii. If the column number $k$ of $\boldsymbol{t}_{1}^{(3)}$ is not zero, put all the columns of $\boldsymbol{t}_{1}^{(3)}$ successively into the $n-1+i(i=1,2, \ldots, k)^{\text {th }}$ column of $\boldsymbol{t}_{1}$, and correspondingly put all columns of $E^{(3)}$ successively into the $n$ $1+i(i=1,2, \ldots, k)^{\text {th }}$ column of $E$. Delete those columns in $\boldsymbol{t}_{1}$ and $E$, on which the lower boundaries of the target function are larger than current $\min E$, update $S_{1}$. After the delete process, if $E$ is nonempty, rearrange the columns of $\boldsymbol{t}_{1}$ and $E$ on ascending order,

f) Output: and turn to a). If $E$ is empty, turn to f).

i. Set $m$ as the column number of $\boldsymbol{L t}$. If $m$ is nonzero, calculate the minimum value of the target function on the elements in $\operatorname{mid}(L t)$, and let the corresponding column No. to be $r$. Therefore, the searching result $\min t_{1}$ is the $\mathrm{r}^{\text {th }}$ column of $\boldsymbol{L} t$. If $\mathrm{m}$ is zero, throw an error message.

ii. Delete those columns in $L t$ and $L E$, on which the lower boundaries of the target function are larger than current $\min E$. Output $L t$ and $L E$. Output the minimum value of the target function $\min E_{1}=\min E$.

iii. Put $\min t_{1}$ into the result vector ResultT.

2) Optimal searching process in sub-feasible-region ${ }^{t_{2}}$ : The procedures are the same with those of $\boldsymbol{t}_{1}$.

M) Optimal searching process in sub-feasible-region $\boldsymbol{t}_{M}$ : The procedures are the same with those of $\boldsymbol{t}_{1}$.

$M+1)$ Check the condition of equation (7). If it is true, quit the searching process and turn to (4); otherwise, continue the iteration and turn to 1);

(4) Calculate the value of the target function on the result vector ResultT, and find the minimum value $\min E$. The column in ResultT would be the final estimation $\min t$.

With the searching result vector ResultT, the system model under the critical fragile status is derived. By detecting the characteristic performance of the critical fragile model, the threshold of the system performance would be clearly concluded. The critical fragile condition would also be the indispensable reference of the maintenance and health monitoring strategies of the whole system.

\section{Experiment}

In this section, a realistic example using the proposed method will be given. The target system is an engineering prototype of an HY-50 DDPC position servo system. Direct drive pump controlled (DDPC) scheme was firstly introduced by Sprockhoff in 1979. The DDPC scheme has been successfully used in various industrial systems, such as systems of plastic injection molding [10], aircraft actuation [11] and ship steering [12]. The structure of this DDPC position servo system is shown in Figure 2. It mainly consists of an $\mathrm{AC}$ rate regulation unit (including servo driver and servo motor MG), a pump actuating unit (including a two-way gear pump, a cylinder, and elements of oil 
circuit: overflow valve RF1, RF2; check valve RCV1, RCV2; hydraulic lock RCV3 etc.), and a computer control unit. The computer control unit integrates required position value and actual position sensor's feedback, then generates the control strategies and sets up a closed-loop control system.

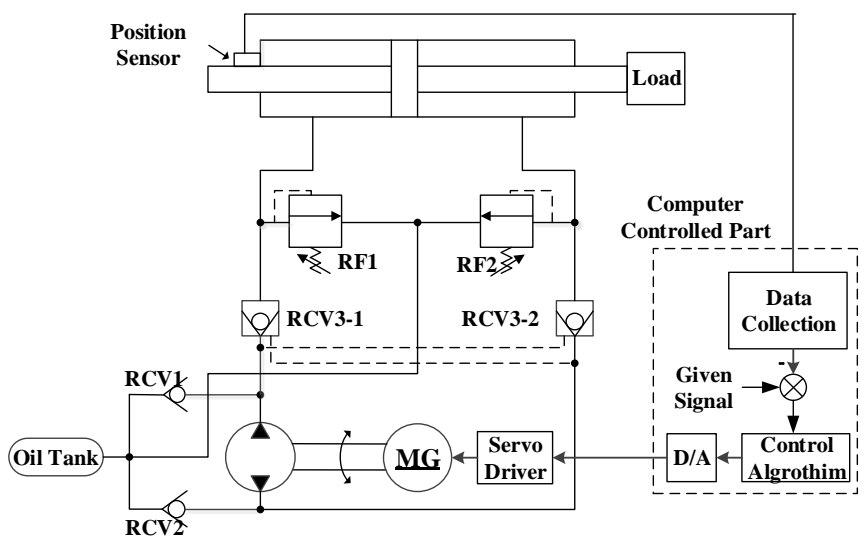

Figure 2. Structure of the HY-50 DDPC Position Servo System

The main block of HY-50 servo system consists of a Panasonic Minas A5 MSME154G1C servo motor, a double way fixed displacement gear pump with a displacement flow of $1.4 \mathrm{~mL} / \mathrm{r}$, a symmetrical hydraulic cylinder with a total movement range of $100 \mathrm{~mm}$, a closed oil tank, a set of valve terminal and other circuit accessories. Figure 3 shows the main block of the HY-50 DDPC servo system. A position sensor and double pressure sensors are also connected to the main block, thus the displacement of the piston and the pressure on both side of the cylinder could be measured. Using data collection board, the position signal is transmitted into the control terminal HY-120, and the control strategies is realized in the control terminal, with a control signal output ranged from $-10 \mathrm{~V} \sim 10 \mathrm{~V}$. The control voltage is then put into the Panasonic Minas A5 MDDHT3420 servo driver, which controls the rotate direction and rate of the servo driver, thus a closed-loop position servo system is formed. Although the scheme and system models of AC rate regulation servo system is quite complicated with some parameters hard to estimate, the high performance $\mathrm{AC}$ rate regulation servo units, which are usually adopted in engineering, have enormously wider response frequency than any other parts in DDPC servo system [13]. For example, the AC rate regulation unit of Panasonic Minas A-series could reach a response frequency as high as $2 \mathrm{kHz}$. Therefore, the AC rate regulation servo unit in DDPC servo system could be simplified as a firstorder inertial system.

With proper assumption, according to flow continuity equation and Newton's Second Law, the transfer function between the piston's position $Y(s)$ and the input voltage of the servo motor $U(s)$ is as (8) when rotation rate $N$ and load $F$ is given [14]

$$
\begin{gathered}
Y(s)=\frac{G_{C}(s) A D_{p} K_{v}}{s\left(\frac{1}{\omega_{n}} s^{2}+2 \frac{\xi_{n}}{\omega_{n}} s+1\right)\left(B_{m} C_{t}+A^{2}\right)\left(T_{e} s+1\right)+K_{s} G_{C}(s) A D_{p} K_{v}} U(s) \\
\omega_{n}=\sqrt{\frac{\beta_{e}\left(B_{m} C_{t}+A^{2}\right)}{V_{1} M_{t}}}, \zeta_{n}=\frac{1}{2}\left(\frac{M_{t} C_{t}+\frac{B_{m} V_{1}}{\beta_{e}}}{B_{m} C_{t}+A^{2}}\right) \omega_{n} .
\end{gathered}
$$

The related parameters are shown is Table 1. 


\section{Table 1. HY-50 DDPC Characteristic Parameters in Nominal}

\begin{tabular}{|l|c|l|}
\hline \multicolumn{1}{|c|}{ Parameters } & Symbols & \multicolumn{1}{c|}{ Values } \\
\hline Rotation rate gain & $K_{v}$ & $5(\mathrm{r} / \mathrm{s}) / \mathrm{V}$ \\
\hline Time constant of servo motor & $T_{e}$ & $0.002 \mathrm{~s}$ \\
\hline Every revolution flow of pump & $D_{p}$ & $1.4 \times 10^{-6} \mathrm{~m}^{3} / \mathrm{r}$ \\
\hline Maximum volume of fluid chamber & $V_{l}$ & $1 \times 10^{-4} \mathrm{~m}^{3}$ \\
\hline Section area of piston side & $A$ & $1.47 \times 10^{-3} \mathrm{~m}^{2}$ \\
\hline Total leakage coefficient of cylinder & $C_{t}$ & $7 \times 10^{-11}\left(\mathrm{~m}^{3} / \mathrm{s}\right) / \mathrm{Pa}$ \\
\hline Effective bulk elastic modulus of oil & $\beta_{e}$ & $7 \times 10^{8} \mathrm{~Pa}$ \\
\hline Equivalent mass of piston & $M_{t}$ & $11 \mathrm{Kg}$ \\
\hline Equivalent viscous damping coefficient & $B_{m}$ & $800 \mathrm{~N} /(\mathrm{m} / \mathrm{s})$ \\
\hline
\end{tabular}

If the $G_{C}(s)$ adopts proportional control scheme, then the order of the scheme model is 4.

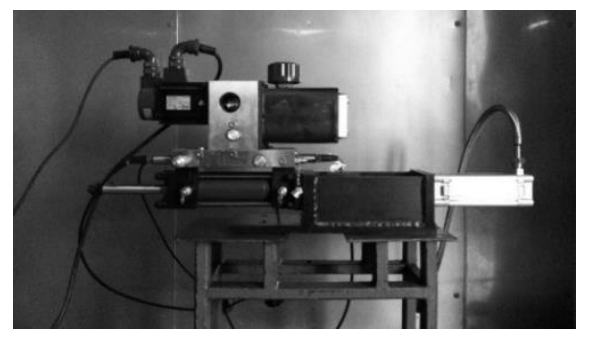

Figure 3. Main Block of HY-50 DDPC Servo System

In order to test the performance of the DDPC position servo system under low temperature condition, the main block of the DDPC system is put into the RGD-1080 high-low temperature test chamber, and the temperature was randomly set to $0^{\circ} \mathrm{C}$, $-15^{\circ} \mathrm{C},-20^{\circ} \mathrm{C}$ and $-30^{\circ} \mathrm{C}$. Step response was tested under those temperatures. The control terminal used a LABVIEW platform to save the test data, and the sampling frequency was set to $10 \mathrm{~Hz}$. With the sampled running data, discrete models under different temperatures could be estimated by least square method, then the corresponding continuous models could be calculated by zero-pole matching method. Table 3 lists the estimated continuous transfer functions, poles of each model, and estimation errors $J$. Due to the complex of the DDPC system, the models cannot be estimated accurately, but the estimation results are generally effective. According to the estimation results shown in Table.2, all the models under low temperatures have two pairs of conjugated poles in the form of $s_{1,2}=a_{1} \pm \mathrm{j} b_{1}, s_{3,4}=a_{2} \pm \mathrm{j} b_{2}, a_{1}, b_{1}, a_{2}, b_{2} \in R$, and the ratio between the numerator and the constant term of denominator is approximately 1 . As the working temperature decreases, the poles generally move towards the imaginary axis. 


\section{Table 2. The Continuous Estimated Models of the DDPC Position Servo System Under Low Temperatures}

\begin{tabular}{|c|c|c|c|}
\hline $\begin{array}{l}\text { Temperature/ } \\
{ }^{\circ} \mathrm{C}\end{array}$ & Continuous estimated model & Poles & $\begin{array}{l}\text { Estimation } \\
\text { error } J\end{array}$ \\
\hline 0 & $G(s)=\frac{1656.3}{s^{4}+13.1 s^{3}+1008.1 s^{2}+2128.4 s+1700.3}$ & $\begin{array}{l}-1.0731 \pm j 0.7605 \\
-5.4996 \pm j 30.8634\end{array}$ & 0.0532 \\
\hline-15 & $G(s)=\frac{908.1302}{s^{4}+11.3876 s^{3}+992.7 s^{2}+1457.9 s+917.5}$ & $\begin{array}{l}-0.7413 \pm \mathrm{j} 0.6241 \\
-4.9525 \pm \mathrm{j} 30.8628\end{array}$ & 0.0667 \\
\hline-20 & $G(s)=\frac{523.5139}{s^{4}+8.7 s^{3}+840.8 s^{2}+1007.2 s+528.4}$ & $\begin{array}{l}-0.6031 \pm j 0.5215 \\
-3.7246 \pm j 28.5879\end{array}$ & 0.0662 \\
\hline-30 & $G(s)=\frac{414.7347}{s^{4}+11.2 s^{3}+1209.3 s^{2}+1268.9 s+417.6}$ & $\begin{array}{l}-0.5280 \pm \mathrm{j} 0.2641 \\
-5.0946 \pm \mathrm{j} 34.2376\end{array}$ & 0.0875 \\
\hline
\end{tabular}

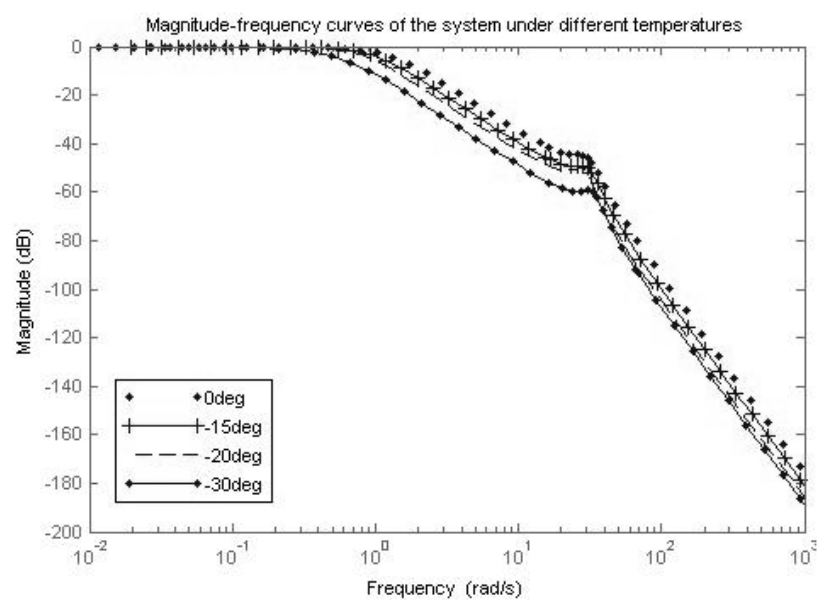

\section{Figure 4. The Magnitude-Frequency Response Curves Under Different Temperatures}

According to Table 2, the magnitude-frequency response curves under different temperatures can be drawn, which is shown in Figure 4. Table 3 shows the $3 \mathrm{~dB}$ bandwidth of these models. As the temperatures decreases, the bandwidth descends, and the response time increases. The nominal lowest working temperature of the servo motor in the HY-50 DDPC servo system is $0^{\circ} \mathrm{C}$. Take a reasonable margin into consideration, the magnitude-frequency threshold at the $3 \mathrm{~dB}$ bandwidth of the model under $0{ }^{\circ} \mathrm{C}$ is chosen as $-6 \mathrm{~dB}$, i.e. $X=6$, and the equivalent power decreases by another $50 \%$ compared with that of the model under $0{ }^{\circ} \mathrm{C}$. Therefore, a unique magnitude-frequency curve can be drawn, since the model is a minimum phase system, the parameters of the critical model can be uniquely determined.

Table 3. 3db Bandwidth of the Models Under Low Temperatures

\begin{tabular}{|c|c|c|c|c|}
\hline Temperature/ ${ }^{\circ} \mathrm{C}$ & 0 & -15 & -20 & -30 \\
\hline Bandwidth $/ \omega_{B}(\mathrm{rad} / \mathrm{s})$ & 1.1177 & 0.8898 & 0.7416 & 0.4311 \\
\hline
\end{tabular}


According to the estimation results, the 4-order transfer function of the critical model can be set as:

$$
G(s)=\frac{\left(a_{1}^{2}+b_{1}^{2}\right)\left(a_{2}^{2}+b_{2}^{2}\right)}{\left(s-a_{1}-\mathrm{j} b_{1}\right)\left(s-a_{1}+\mathrm{j} b_{1}\right)\left(s-a_{2}-\mathrm{j} b_{2}\right)\left(s-a_{2}+\mathrm{j} b_{2}\right)}
$$

Set the magnitude response at $\omega$ as $20 \lg |G(\mathrm{j} \omega)|$, the bandwidth under nominal worst working condition as $\omega_{B 0}$, therefore the parameter estimation of the critical model turns into the following optimization problem:

$$
\begin{aligned}
& \min E=|20 \lg | G\left(\mathrm{j} \omega_{B 0}\right)|+6| \\
& \text { s.t. } a_{1} \in S_{1}, b_{1} \in S_{2}, a_{2} \in S_{3}, b_{2} \in S_{4}
\end{aligned}
$$

, where $S_{1}, S_{2}, S_{3}, S_{4}$ are the feasible regions of $a_{1}, b_{1}, a_{2}, b_{2}$ respectively. Basing on the supposed conditions, the magnitude $-6 \mathrm{~dB}$ on frequency $\omega_{B 0}$ lies between the magnitudefrequency response curves of $-15{ }^{\circ} \mathrm{C}$ and $-20{ }^{\circ} \mathrm{C}$, therefore the feasible regions of $a_{1}, b_{1}, a_{2}, b_{2}$ are:

$$
\begin{aligned}
& S_{1}=[-0.7413,-0.6031], S_{2}=[0.5215,0.6241], S_{3}=[-4.9525,-3.7246], S_{4}=[28.5879,30.8628] \\
& a_{1} \in S_{1}, b_{1} \in S_{2}, a_{2} \in S_{3}, b_{2} \in S_{4}
\end{aligned}
$$

Since the model has two pairs of conjugate poles, the estimating parameters can be divided into two groups, one group is $a_{1}, b_{1}$, and the other is $a_{2}, b_{2}$. Correspondingly, the sub-feasible-region can be iterated as follows:

$$
\begin{aligned}
& t_{1}=\left[S_{1} ; S_{2} ;\left[\operatorname{mid}\left(\min t_{2}(3)\right), \operatorname{mid}\left(\min t_{2}(3)\right)\right] ;\left[\operatorname{mid}\left(\min t_{2}(4)\right), \operatorname{mid}\left(\min t_{2}(4)\right)\right]\right]^{\mathrm{T}} \\
& t_{2}=\left[\left[\operatorname{mid}\left(\min t_{1}(1)\right), \operatorname{mid}\left(\min t_{1}(1)\right)\right] ;\left[\operatorname{mid}\left(\min t_{1}(2)\right), \operatorname{mid}\left(\min t_{1}(2)\right)\right] ; S_{3} ; S_{4}\right]^{\mathrm{T}}
\end{aligned}
$$

Interval computing in this paper is realized by INTLAB kit [15] developed by Institute for Reliable Computing in Hamburg University of Technology. On a computer platform of Core 2 Duo E7300 2.6 GHz with 2GB RAM, the multi-group iterative interval branch-and-bound algorithm was realized in MATLAB 2012b. The operation time was approximately $11 \mathrm{~s}$, which is much faster than the time consumed by searching for four parameters simultaneously. When the width of interval elements of min $t$ is less than 0.01 , the algorithm terminates. The searching results are:

$$
\begin{aligned}
& \min t=\left[\begin{array}{c}
{[-0.6170,-0.6169]} \\
{[0.5686,0.5687]} \\
{[-3.7345,-3.7246]} \\
{[28.5879,28.5916]}
\end{array}\right] \\
& \min E=0.0011
\end{aligned}
$$

To select conservatively fixed values from the interval elements in mint: choose the lower boundary of the $1^{\text {st }}$ and $3^{\text {rd }}$ element as $a_{1}, a_{2}$, which is far from the imaginary axis; choose the middle value of the $2^{\text {nd }}$ and $4^{\text {th }}$ element as $b_{1}, b_{2}$. Therefore, the critical model is:

$$
\begin{aligned}
G(s)= & \frac{585.2524}{(s+0.6170-\mathrm{j} 0.56865)(s+0.6170+\mathrm{j} 0.56865)} \\
& \cdot \frac{1}{(s+3.7345-\mathrm{j} 28.5897)(s+3.7345+\mathrm{j} 28.5897)}
\end{aligned}
$$


According to critical model (13), the critical magnitude-frequency response curve and step response curve could be drawn, which are shown in Figure 5. It could be seen that the $3 \mathrm{~dB}$ bandwidth of the critical model is $0.8053 \mathrm{rad} / \mathrm{s}$, and the settling time of $100 \mathrm{~mm}$ step response is approximately $8 \mathrm{~s}$.

With the critical model and its characteristics, a quick and efficient fragile status evaluation could be made to the DDPC servo system:

(1) If the $3 \mathrm{~dB}$-bandwidth of the system is equal to or lower than the critical model, or the settling time of the system is equal to or longer than the critical model, it can be considered that the system operates under the critical fragile status. If the system keeps working on these status, potential faults might occur.

(2) Under low temperature condition, for HY-50 DDPC servo system, the critical fragile operating temperature lies between $-15^{\circ} \mathrm{C}$ and $-20^{\circ} \mathrm{C}$. So once the operating temperature drops to $-15^{\circ} \mathrm{C}$, or the system needs to operate under harsh temperatures, special attention and measures should be taken to prevent abnormal status or faults from occurring. Actually, running noises occurred under short time experiments below $-20^{\circ} \mathrm{C}$.

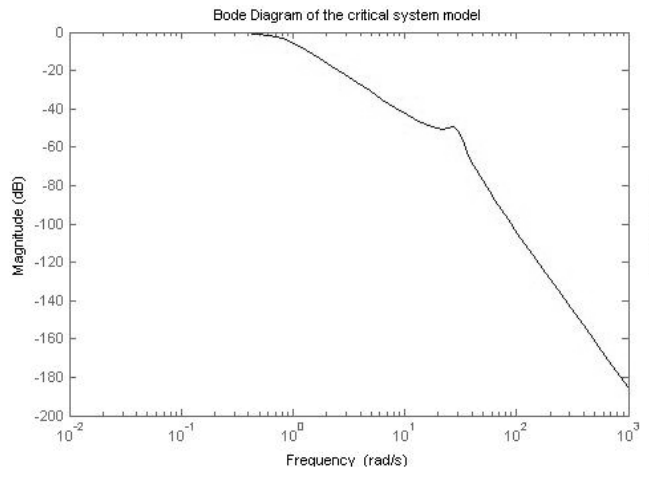

(a)

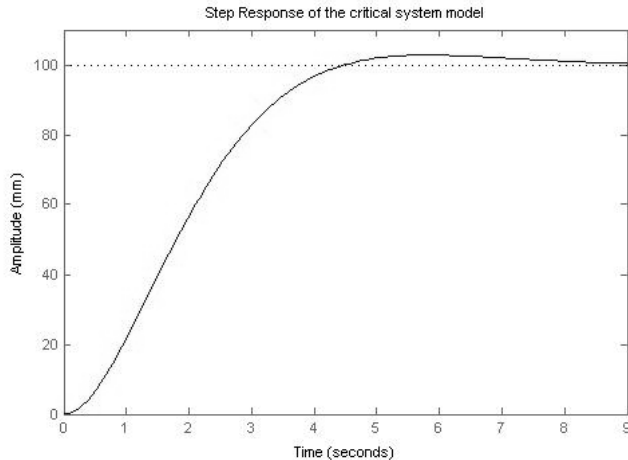

(b)

Figure 5. The Critical Model's: (a) Magnitude-Frequency Response Curve (b) $100 \mathrm{~mm}$ Step Response Curve

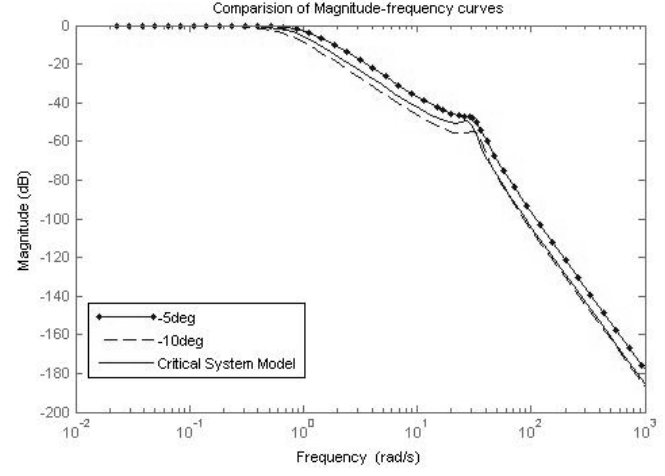

(a)

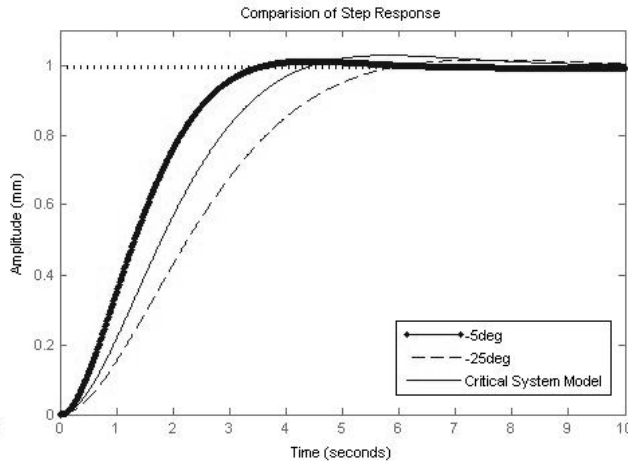

(b)

Figure 6. (a) Comparison of Magnitude-Frequency Curve between the System Under $-5^{\circ} \mathrm{C}$ and $-25^{\circ} \mathrm{C}$ and the Critical Model (b) Comparison of Step Response Between the System Under $-5^{\circ} \mathrm{C}$ and $-25^{\circ} \mathrm{C}$ and the Critical Model 
According to the results, step response tests were carried under the temperature of $-5^{\circ} \mathrm{C}$ and $-25^{\circ} \mathrm{C}$. Compared to the performance characteristics of the critical model, the bandwidth of the system under $-5^{\circ} \mathrm{C}$ was better, and the settling time of the system under $-5^{\circ} \mathrm{C}$ was less; while under $-25^{\circ} \mathrm{C}$, the bandwidth of the system was worse than the critical model, and the settling time was longer than the critical model. It could also be easily seen from Figure 6 , that the system under $-5^{\circ} \mathrm{C}$ could fulfill the performance requirements while the system under $-25^{\circ} \mathrm{C}$ could not. Therefore, after establishing the critical model, the performance and fragile status of the system under different operation conditions could be easily and fast evaluated by testing the magnitude-frequency response and step response and comparing with the characteristics of the critical model.

\section{Conclusions}

Fragility is an intrinsic characteristic of the compromising-designed industrial electromechanical systems, and it could result in the performance degradation and possible failure in harsh operating conditions. Due to the mature schemes and the acceptable approximation in realistic, many of the industrial systems could be simplified into linear minimum phase system. This paper takes advantages of the interval model of the system under harsh operating conditions, and uses the stable margin to judge the critical fragile status. The order of the system model is determined by the system scheme; the interval model of the system under the specified range of harsh operating conditions with the aid of the least square method and the corresponding operation data; the criterion of the critical fragile status of the system is defined as a power drop amount; a sub-optimal multi-group iterative interval branch-and-bound algorithm is proposed to find the critical fragile model of the system. The experiment and analysis of the DDPC servo system prototype shows the validity of the proposed method. In practical, the critical model could be established by a series of simple tests. Thereafter, with aid of online monitoring facilities such as position transducer and frequency analyzer, the fragile status servo system could be evaluated in time, so that proper counter measurements could then be quickly adopted to cope with the potential hazards and possible failures. The proposed method has contribution for the performance evaluation and fragility analysis of electromechanical systems with clear schemes.

It should be mentioned that the noise in data logging largely affects the precision of the critical fragile model. It still needs further research to deal with the issues of model precision and the situations with mixed causes of performance degradation and fragility.

\section{Acknowledgements}

The authors would like to thank the support of Shanghai Science and Technology Committee under Grant No. 11JC1413000.

\section{References}

[1] L. H. Keel and S. P. Bhattacharyya. 'Robust, fragile, or optimal?', IEEE Transactions on Automatic Control, vol. 42, no. 8, (1997), pp. 1098-1105.

[2] P. M. Mäkilä. 'Comments on "Robust, fragile, or optimal?"', IEEE Transactions on Automatic Control, vol. 43, no. 9, (1998), pp. 1265-1268.

[3] G. X. Wang, J. Zhang and J. Q. Zhu. 'Output feedback control of inverted pendulum: fragility and robustness', Electric Machines and Control, vol. 6, no. 3, (2002), pp. 221-223.

[4] Z. He, D. Rao, G. X. Wang and Z. Y. Liu. 'Fragility and robustness of the control systems', Electric Machines and Control, vol. 15, no. 4, (2011), pp. 80-84.

[5] Z. He, G. X. Wang, J. Zhang, J. C. Qi and D. Zhou. 'Fragility analysis for control systems', Control Theory \& Applications, vol. 30, no. 1, (2013), pp. 95-100.

[6] R. E. Moore. Interval analysis, Englewood Cliffs: Prentice-Hall, (1966), pp. 1-195. 
[7] P. B. Li, D. W. Hu, J. Y. Zhang and H. W. Xie. System Identification, Beijing: China Water Power Press, (2010), pp. 55-61.

[8] H. Tuy and R. Horst. 'Convergence and restart in branch-and-bound algorithm for global optimization application to concave minimization and D.C. optimization problems', Mathematical Programming, vol. 41, (1989), pp. 161-183.

[9] Y. M. Wang, J. G. Yue, Y. Q. Su and H. Liu. 'Design of two-dimensional zero-phase FIR digital filter by McClellan transformation and interval global optimization', IEEE Transactions on Circuits and Systems II- Express Briefs, vol. 60, no. 3, (2013), pp. 167-171.

[10] Bosch AG. 'Electro-hydraulic proportional radial piston pump direct integrated into the closed loop system', O+P, vol. 39, no.11-12, (1995), pp. 819-821.

[11] M. Glauss. 'Hydraulic proportional pump controlled with industrial bus and integrated digital on-board electronic circuit', O+P, vol. 44, no. 9, (2000), pp. 552-556.

[12] H. Sato. 'The structure and the application of the electro-hydraulic control system without valves and pipes', Hydraulic and Pneumatic Technology, vol. 6, (2001), pp. 36-42.

[13] H. B. Wang, Y. S. Zhang, S. C. Feng and D. R. Gao. 'System modeling and adaptive back-stepping sliding mode control for direct-drive pump-controlled cylinder', Electric Machines and Control, vol. 15, no. 9, (2011), pp. 92-97.

[14] M. Y. Cao, Q. Sun, H. Guo and J. G. Yue. 'Modeling and simulation of direct drive volume electrohydraulic servo system', Chinese Hydraulics and Pneumatics, no.5, (2013), pp. 41-44.

[15] S. M. Rump. INTLAB - INTerval LABoratory. Developments in Reliable Computing, Kluwer Academic Publishers, Dordrecht, (1999), pp. 77-104.

\section{Authors}

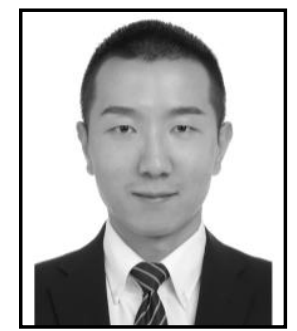

Qiang Sun, is currently a $\mathrm{PhD}$ candidate at Tongji University, China. His research interests include fault prognosis and reliability analysis.

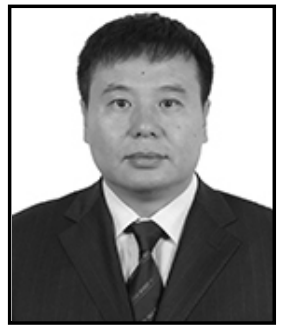

Jiguang Yue, is currently a professor and a $\mathrm{PhD}$ candidate supervisor at Tongji University, China. His research interests include fault prognosis and advanced process control

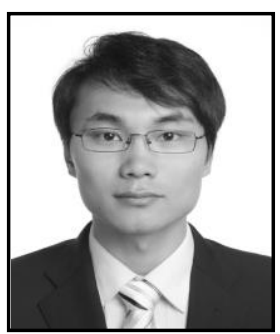

Yanming Wang, is currently a $\mathrm{PhD}$ candidate at Tongji University, China. His research interests include interval arithmetic and machine vision.

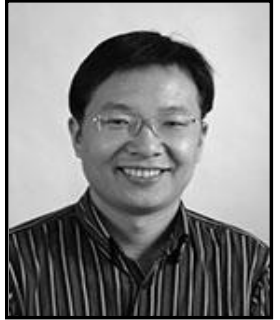

Yongqing Su is currently an associate professor at Tongji University, China. His research interests include interval arithmetic and advanced process control. 


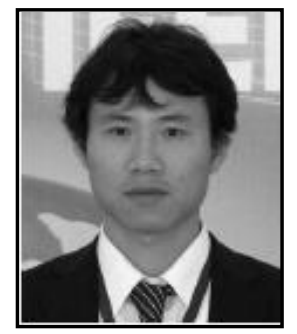

Yanchao Dong, is currently a lecturer at Tongji University, China. His research interests include interval arithmetic and machine vision. 